infusion. Our last meeting in July 1990 was such an occasion and the last physics we talked about was F.J. Dyson's paper "Feynman's Proof of the Maxwell Equations" [23]. Like his many other friends, I shall miss these informal discussions.

\section{REFERENCES}

[1] Bell J.S., Speakable and Unspeakable in Quantum Mechanics (Cambridge University Press, Cambridge) 1987.

[2] See e.g. Bernstein J., Quantum Profiles (Princeton University Press, Princeton) 1991.

[3] Bell J.S., "On Problems of Hidden Variables in Quantum Mechanics", Rev. Mod. Phys. 38 (1966) 447.

[4] Bell J.S., " On the Einstein-Rosen-Podolsky Paradox", Physics 1 (1964) 195.

[5] Bell J.S., "Against 'Measurement", Physics World 3 (1990) 33

[6] Bell J.S. and Jackiw R., "A PCAC Puzzle: $\pi^{\circ}-2 \gamma$ in the $\sigma$-model", Nuovo Cimento A60 (1969) 47.

[7] Bell J.S. and Leinaas J.M., "Electrons as Accelerated Thermometers", Nucl. Phys. B212 (1983) 131; "The Unruh Effect and Quantum Fluctuations of Electrons in Storage Rings", Nucl. Phys. B284 (1987) 488. [8] Bell J.S., "Time Reversal in Field Theory", Proc. Roy. Soc. A231 (1955) 479.
[9] Bell J.S., Hughes, R.J. and Leinaas J.M., "The Unruh Effect in Extended Thermometers", Z. Phys. C28 (1985) 75.

[10] Bell M.A. and Bell J.S., "Quantum Bremsstrahlung", Part. Accel. 22 (1988) 301; " End Effects in Quantum Bremsstrahlung", Part. Accel. 24 (1988) 1; "Quantum Bremsstrahlung in Almost Uniform Fields", Nucl. Inst. and Methods A275 (1989) 258. [11] Bell J.S., "Hamiltonian Mechanics": in Proc. CERN Accelerator School: Advanced Accelerator Physics, Ed. S. Turner, CERN 87-03 (Vol. I) 1987.

[12] Bell J.S., "How to Teach Special Relativity", Prog. in Scientific Culture 1 No. 2 (1976); reprinted in [1].

[13] Bell J.S. and Hey A.J.G., "A Theoretical Argument for Something Like the Second Melosh Transformation", Phys. Lett. B51 (1974) 365.

[14] Bell J.S. and Ruegg H., "Hydrogen Atom on Null Plane and Melosh Transformation", Nucl. Phys. B93 (1975) 12; "Positronium on Null Plane and Melosh Transformation", Nucl. Phys. B104 (1976) 245

[15] Bell J.S. and Hey A.J.G., "Partons of a One-Dimensional Box", Phys. Lett. B74 (1978) 77; Bell J.S., Davies A.C. and Rafelski J., "Partons of a Spherical Box", Phys. Lett. B78 (1978) 67.

[16] Bell J.S., "Gravity" in Fundamental Systems, Eds. P. Bloch, P. Pavlopoulos and R. Klapisch (Plenum, New York) 1987.
[17] Bell J.S. and Steinberger J., "Weak Interactions of Kaons", Proc. Oxford Int. Conf. on Elementary Particle Physics (1965); reprinted in CP Violation, Ed. L. Wolfenstein (North-Holland, Amsterdam) 1989. [18] Bell J.S. and Perring J.K., " $2 \pi$ Decay of the $\mathrm{K}_{2}^{\circ}$ Meson", Phys. Rev. Lett. 13 (1964) 348.

[19] Bell J.S. and BertImann R.A., "Magic Moments", Nucl. Phys. B177 (1981) 218 "Shifman-Vainshtein-Zakharov Moments and Quark-Antiquark Potentials", Nucl. Phys. B187 (1981) 285; "Gluon Condensate Potentials", Nucl. Phys. B227 (1983) 435; "SVZ Moments for Charmonium and Potential Models", Phys. Lett. B137 (1984) 107.

[20] Bell J.S., "Bertlmann's Socks and the Nature of Physical Reality", J. Phys., Coll. C2, 42 (1981) C2; reprinted in [1].

[21] Rajaraman R. and Bell J.S., "On Solitons with Half-Integer Charge", Phys. Lett. B116 (1982) 115; "On States, on a Lattice, with Half-Integer Charge", Nucl. Phys. B220 (1983) 1.

[22] Bell J.S. and Llewellyn Smith C.H., "Near-Forward Neutrino Reactions on Nuclear Targets", Nucl. Phys. B24 (1970) 285; "Quasielastic Neutrino-Nucleus Interactions", Nucl. Phys. B28 (1971) 317.

[23] Dyson F.J., "Feynman's Proof of the Maxwell Equations", Am. J. Phys. 58 (1990) 209.

\title{
John Bell and Accelerator Physics
}

\section{Mary Bell \\ Geneva, Switzerland}

I shall mention some of John's early work on accelerator physics that is often forgotten because of the great interest in his more romantic papers on the foundations of quantum mechanics.

I first saw John in 1949 soon after his arrival at the Atomic Energy Research Establishment, Harwell, UK when he was 21 years old. He worked there on reactors for about two months before being sent to Malvern to join the very small, theoretical accelerator group run by Bill Walkinshaw that had developed out of wartime work on radar. He lived at Malvern in the Geraldine Hostel with a group of young colleagues who were all keen on motorcycles, which they regularly took to bits and reassembled.

The association with Bill Walkinshaw was a very happy and productive one. Both had a wide knowledge of electromagnetic theory and Bill did not mind John's Celtic temperament. They wrote together several papers on disc-loaded and dielectric-loaded electron accelerators (some of the former were built at that time for medical purposes). The mathe-

Mary Bell, the wife of John Bell, retired recently from CERN, Geneva where she had been an accelerator physicist.
Mary and John Bell taken by Mrs Bertlmann, in Vienna in 1984.
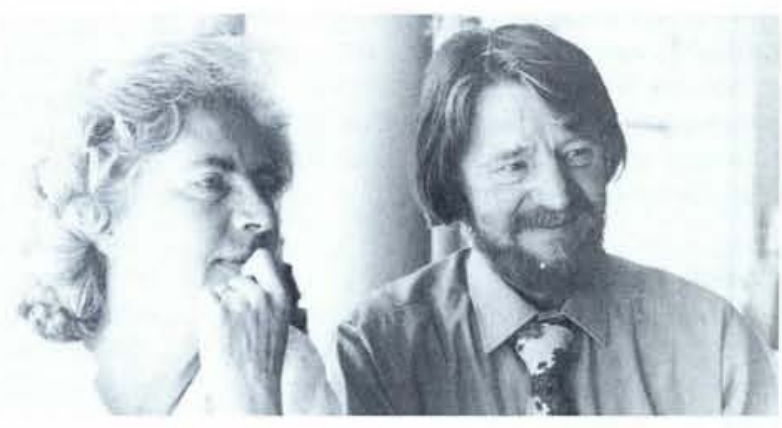

matical techniques which were developed allowed the guides to be designed using a moderate amount of calculation done on a desk calculator - this being before large computers were available.

Later, when CERN was being set up, he attended several preliminary meetings in Geneva with Bill. When the strong-focussing principle appeared, he derived what became known as the Courant-Snyder invariant. Owing to his thorough knowledge of classical mechanics, he was able on several occasions to see that certain results were wrong because they disagreed with general principles. For example, a non-Hamiltonian treatment of accelerating gaps introduced by W.K.H.. Panofsky can produce incorrect results. His work in this field is often unrecognized, with reviewers simply referring to "unpublished" Harwell reports.

Although John left the accelerator field after three years to go to Birmingham to work with Professor R. Peierls (the second - after Henry Flowers - to take part in Harwell's university scheme) he always retained an interest in the area. I was to collaborate with him on some papers on electron cooling in the preparation for CERN's Super Proton Synchrotron when I was with Frank Krienen's group. Although the alternative technique of stochastic cooling was found to be cheaper, electron cooling was eventually used for the LEAR ring. We also collaborated on bremsstrahlung in studies of linear colliders [this page, 10].

John remained very practical and was no vague theorist. I believe he even attended a course for bricklayers as a student at the Technical College in Belfast. He could always put numbers to a problem. He once laughed on finding $A$ Textbook of Physics by N. Watson on my desk, but it was my turn to be amused many years later when I saw him using the same book, with its many tables of materials' properties, to work out where our refrigerator should be placed. Indeed, he remarked at the time that he would have liked to have been a modern Watson. 